\title{
Detection of lean blowout in a premixed gas-turbine model combustor based on nonlinear dynamics
}

\author{
Y. Shinoda ${ }^{1}$, M. Kobayashi ${ }^{1}$, H. Gotoda ${ }^{1}$ \& S. Tachibana ${ }^{2}$ \\ ${ }^{I}$ Department of Mechanical Engineering, Ritsumeikan University, \\ Shiga, Japan \\ ${ }^{2}$ Aerospace Research and Development Directorate, \\ Japan Aerospace Exploration Agency, Tokyo, Japan
}

\begin{abstract}
A detection method of lean blowout in a laboratory-scale premixed gas-turbine model combustor has been proposed from a view point of nonlinear dynamics. The translation error, which quantifies the degree of the parallelism of trajectories in the phase space constructed from the time-series data of the combustion-chamber pressure fluctuations, is used as a detector in this work. The dynamic behavior in the complex combustion instability close to lean blowout is successfully characterized by the translation error. The obtained results show that the translation error has a potential use for detecting the lean blowout in a lean premixed gas-turbine combustor.

Keywords: lean premixed gas-turbine combustor, lean blowout, detection, nonlinear dynamics.
\end{abstract}

\section{Introduction}

Lean premixed combustion is highly advantageous for reducing nitrogen oxide $\left(\mathrm{NO}_{\mathrm{x}}\right)$ emission from gas-turbine engines without the loss of combustion efficiency by controlling the equivalence ratio to within an appropriate range. This combustion method has attracted considerable attention from developers of gas-turbine combustors. A major drawback of lean premixed combustors, however, is that they are susceptible to flow perturbations. They suffer from combustion instabilities such as thermoacoustic combustion oscillations, lean 
blowout and flashback. Thermoacoustic combustion instability, caused by the strong coupling between the variations in pressure and heat-release rate, is considered to be a serious problem since it can lead to a reduction in the lifetime or even the total destruction of an engine. The physical mechanism underlying the onset of thermoacoustic combustion instability and efficient methods of suppressing the combustion instability have been extensively investigated for various types of laboratory-scale gas-turbine combustor with swirling flow, which were summarized in detail in a recent review paper edited by Huang and Yang [1]. Regarding the treatment of the dynamic behavior of the thermoacoustic combustion instability, power spectral analysis of the pressure and heat-release fluctuations has been performed in most studies [1-9]. This method has been widely used to characterize unstable combustion modes, but may be insufficient for understanding and interpreting the physics underlying the combustion instability because it is a complex phenomenon strongly affected by the inherent nonlinearity associated with chemical reactions, turbulent flow and acoustic perturbations. Therefore, in addition to conventional methods of linear analysis such as power spectrum analysis, a new approach based on nonlinear dynamics will be required for characterizing the complex combustion instability in lean premixed gas-turbine combustors.

The nonlinear time series approach inspired by chaos theory conveys useful information for clarifying the nonlinear properties of complex dynamics, and its utilization has led to the possibility of uncovering information about the underlying dynamics of flame or combustion instability. In recent years it has been applied to the temporal behavior of the unstable combustion state observed in experiments (e.g., a thermal pulse combustor [10], a spark ignition engine $[11,12]$ and swirling premixed flames [13]). The characteristics of the combustion dynamics in gas-turbine combustors have been discussed by Lieuwen [6], Kabiraj et al. [14] and Noble et al. [15] from the viewpoint of nonlinear dynamics. In these works $[6,14,15]$, a method of inferring the correlation dimension (a type of fractal dimension) of trajectories in phase space, that is, the Grassberger-Procaccia (GP) method [16], has been used to quantify the nonlinear characteristics of complex dynamic behavior. The GP algorithm is one of the classic methods of nonlinear time series analysis but has often been reported to lead to misdiagnosis in characterizing the dynamic properties of observational time series. Without using the correlation dimension, two of the current authors [17] have recently reported the possibility that the dynamic behavior of the intermittent oscillation is low-dimensional chaotic, by estimating the translation error that is a method of measuring the parallelism of trajectories in the phase space. In addition, the nonlinear forecasting method based on orbital instability in phase space was also shown to be useful for predicting the short-term dynamic behavior of the intermittent oscillations from a practical viewpoint. The importance of the nonlinear time series analysis to the treatment of the thermoacoustic combustion oscillations has been shown, but there have been no attempts to detect the lean blowout from the viewpoint of nonlinear dynamics. The early detection of the lean blowout is of practical importance to the present-day development of the combustion system. 
The purpose of this study is to propose a detection method of lean blowout in a laboratory-scale premixed gas-turbine model combustor from a view point of nonlinear dynamics. The translation error, which quantifies the degree of the parallelism of trajectories in the phase space constructed from the time-series data of the combustion-chamber pressure fluctuations, is used as a detector in this work.

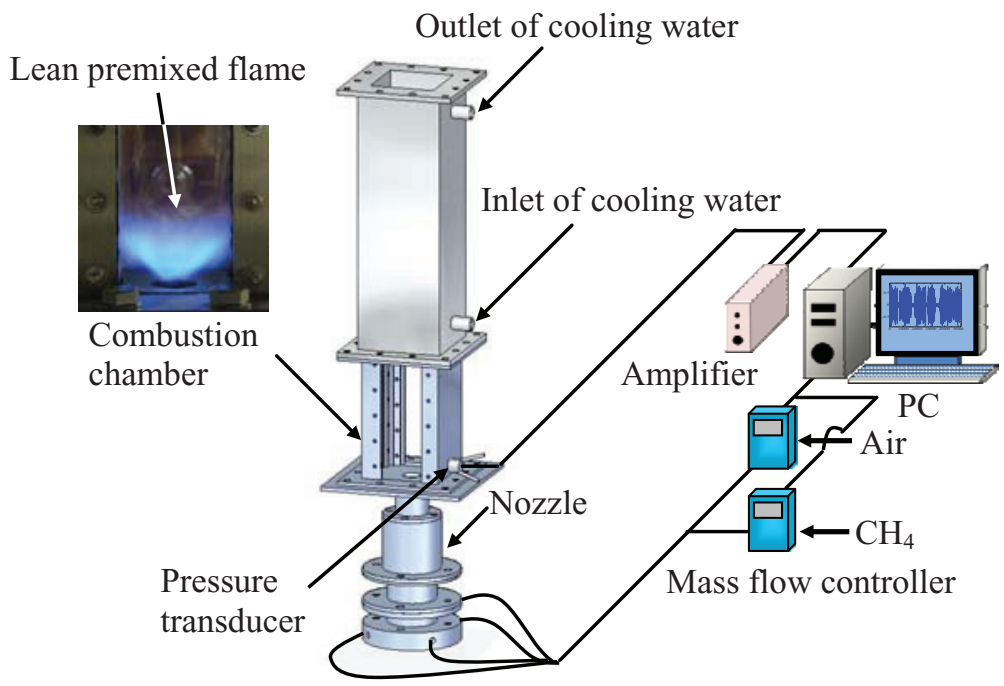

Figure 1: Experimental system.

\section{Experimental apparatus and method}

The configuration of the experimental system is shown in Fig. 2. The combustion test rig is composed of a mass flow controller, a nozzle, an axial swirler and a combustion chamber. The chamber has a length of $620 \mathrm{~mm}$ with a $100 \times 100 \mathrm{~mm}$ square cross section. The rest of the chamber is composed of a water-cooled stainless-steel duct. Methane gas is used for the fuel. An axial swirler is installed as a flame holder at the inlet of the combustion chamber.

The inlet air temperature and volume flow rate (mean axial flow velocity at inlet of combustion chamber) are $300 \mathrm{~K}$ and $148 \mathrm{~L} / \mathrm{min}(3.5 \mathrm{~m} / \mathrm{s})$, respectively. The equivalence ratio $\phi$ is varied from 0.45 to 0.70 . The pressure fluctuations are measured by pressure transducers (JTEKT Products, PD104K-10kPa). A pressure port is located at $20 \mathrm{~mm}$ from the inlet of combustion chamber on the wall of the combustion chamber. Signals from the pressure transducers are acquired through a data acquisition system (LabVIEW system, National Instruments). The nonlinear time series analysis is conducted for the time series data of the pressure fluctuations $p^{\prime}$ at the sampling frequency of $5 \mathrm{kHz}$. 

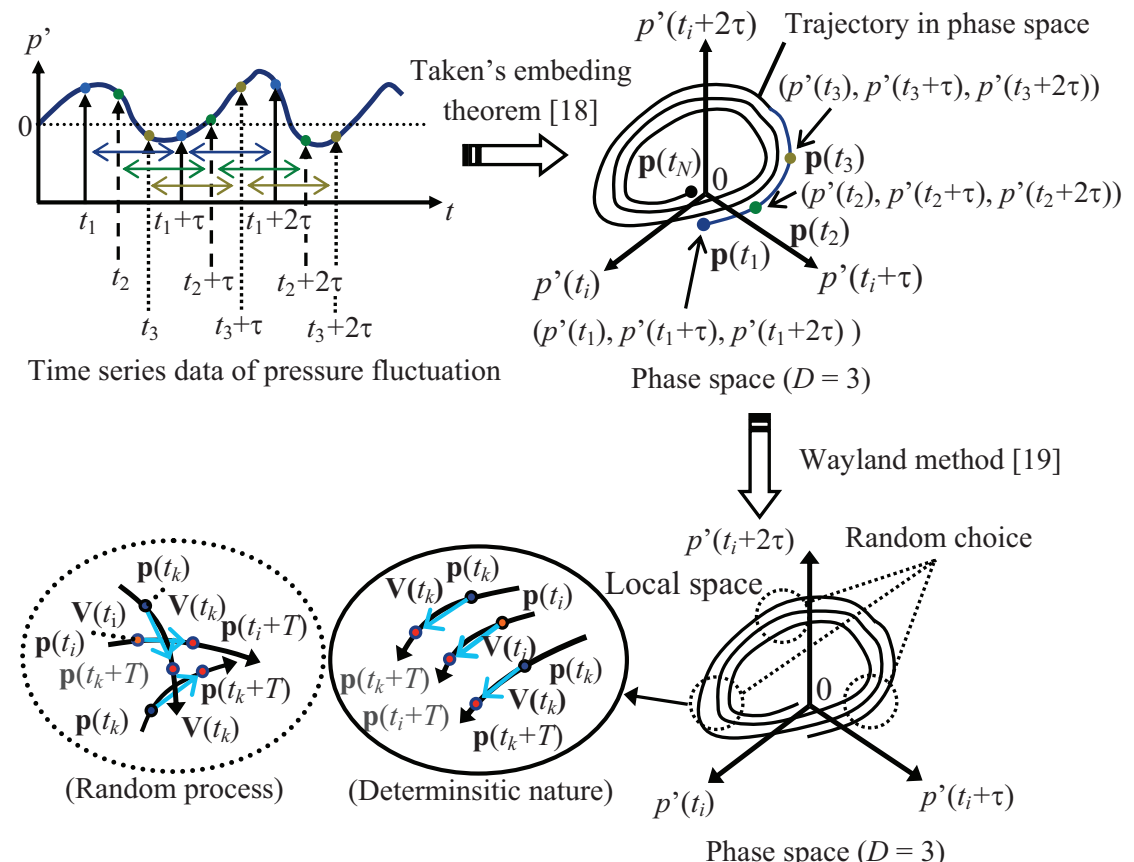

Figure 2: $\quad$ Nonlinear time series analysis.

\section{Nonlinear time series analysis}

The central ideas behind mathematics of the nonlinear time series analysis employed in this work are briefly described as follows. On the basis of Takens' embedding theorem [18], the phase space is constructed from the time series data of the pressure fluctuations $p^{\prime}$ as shown in Fig. 2. The time-delayed coordinates used for the construction of the phase space are expressed as

$$
\mathbf{p}\left(t_{i}\right)=\left(p^{\prime}(t), p^{\prime}(t+\tau), p^{\prime}(t+2 \tau), \ldots, p^{\prime}(t+(D+1) \tau)\right)
$$

where $i=0,1, \cdots, n$ ( $n$ is the data number of the time series), $\mathbf{p}\left(t_{i}\right)$ are the phase space vectors, $p^{\prime}(t)$ are the pressure fluctuations at time $t_{i}, D$ is the embedding dimension, that is, the dimension of the phase space, and $\tau$ is a time lag. If the time lag is too small, then the elements of the phase space vectors are strongly correlated. If the time lag is too large, then the correlation is lost completely. As reported in a previous work [17], an appropriate choice for the time lag is made using mutual information.

The neighboring trajectories in the phase space have similar directions if the determinism is visible in a time series. On the basis of this concept, we introduce a useful algorithm proposed by Wayland et al. [19] to extract the nonlinear determinism in a time series data. Let us randomly select a vector $\mathbf{p}\left(t_{i}\right)$ 
and find its $K$ nearest neighbors $\mathbf{p}\left(t_{k}\right)$ with $k$ from 1 to $K$. In this work, the value of $K$ is set to 10 . We set the images of all the vectors to $\mathbf{p}\left(t_{k}+T\right)$ at a suitable time interval $T$. The diversity in the directions of neighboring trajectories is measured in terms of the translation error $E_{\text {trans }}$, defined by

$$
\begin{gathered}
E_{\text {trans }}=\frac{1}{K+1} \sum_{k=0}^{K} \frac{\left\|\mathbf{V}\left(t_{k}\right)-\overline{\mathbf{V}}\right\|}{\|\overline{\mathbf{V}}\|} \\
\overline{\mathbf{V}}=\frac{1}{K+1} \sum_{k=0}^{K} \mathbf{V}\left(t_{k}\right) \\
\mathbf{V}\left(t_{k}\right)=\mathbf{p}\left(t_{k}+T\right)-\mathbf{p}\left(t_{k}\right)
\end{gathered}
$$

In this work, the value of $T$ is set to 8 . Here, $\mathbf{V}\left(t_{k}\right)$ approximates the tangential vectors of trajectories at time $t_{k}$. The more parallel trajectories are to each other, that is, the more visible determinism there is in the time series, the closer $E_{\text {trans }}$ will be to zero. In estimating the translation error, we can reduce the stochastic error by obtaining the medians of $E_{\text {trans }}$ for $Q$ sets of $M$ randomly chosen $\mathbf{p}\left(t_{i}\right)$ and then taking the mean of the $Q$ medians. In this work, the values of $M$ and $Q$ are set to 100 . In this work, $E_{\text {trans }}$ is obtained every $100 \mathrm{~ms}$.

\section{Results and discussion}

The time variations of the pressure fluctuations $p$ and the translation error $E_{\text {trans }}$ of neighboring trajectories in the phase space for $\phi=0.65$ is shown in Fig. 3 . The intermittency of the pressure fluctuations is clearly observed at $t \approx 14.5$ and $27.3 \mathrm{sec}$. When the amplitude of $p$ ' drastically changes by the onset of the intermittency, the value of $E_{\text {trans }}$ also changes simultaneously. The instantaneous response of the translation error to the drastic change in the pressure fluctuations indicates that the translation error is feasible for characterizing the combustion state in a lean premixed gas-turbine model combustor. The translation error $E_{\text {trans }}$ of neighbouring trajectories in the phase space is shown in Fig. 4 as a function of the equivalence ratio $\phi$. When $\phi$ is 0.70 , the value of $E_{\text {trans }}$ is approximately 0.002 , which shows that the determinism in dynamic behaviour of the pressure fluctuations strongly exists. $E_{\text {trans }}$ gradually increases with decreasing $\phi$, but drastically increases when the intermittency of the pressure fluctuations occurs. With a further decrease in $\phi$, it monotonically increases and the determinism in dynamic behaviour of the pressure fluctuations is lost due to the increase in the degree of complexity in the combustion instability. Our recent work of a different type of lean premixed gas-turbine model combustor has clearly shown that the dynamic behaviour of the pressure fluctuations becomes complicated near lean blowout by sophisticated nonlinear time series analysis involving the permutation entropy in combination with surrogate data method, the multifractal analysis and nonlinear forecasting based on a neural network [20]. The determinism in the dynamic behaviour of the pressure fluctuations is lost as the combustion state leads to lean blowout. However, as 


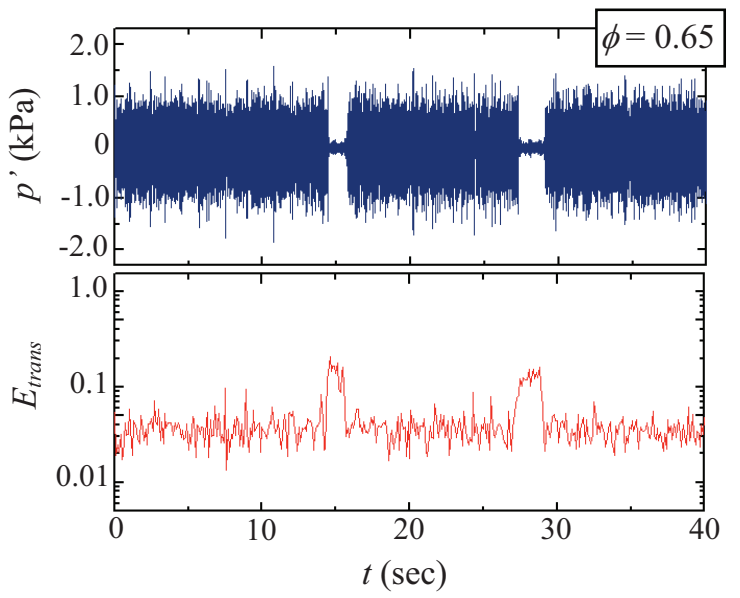

Figure 3: $\quad$ Time variation in $p^{\prime}$ and $E_{\text {trans }}$ at $\phi=0.65$.

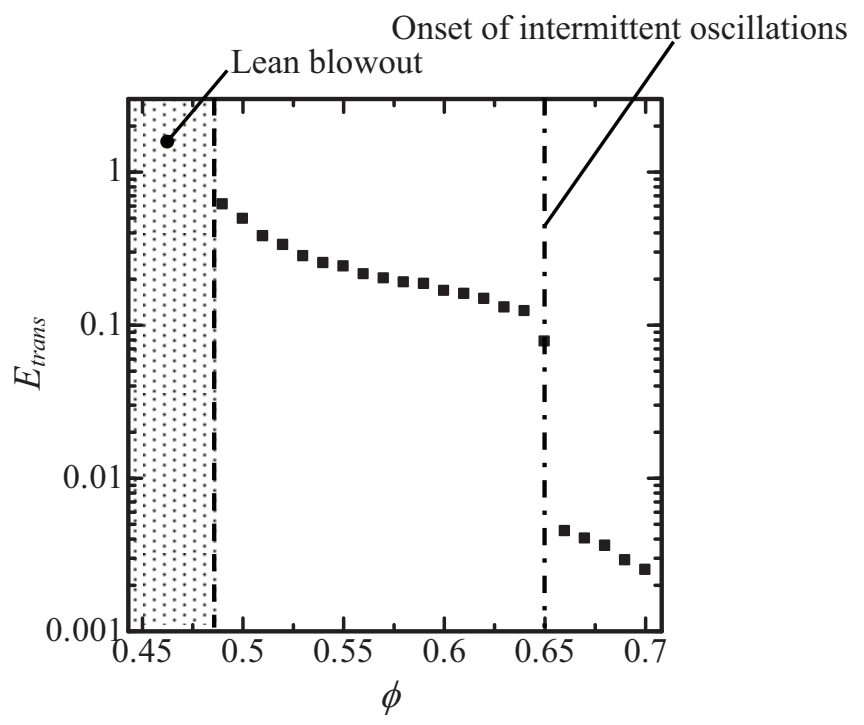

Figure 4: $\quad E_{\text {trans }}$ as a function of $\phi$.

shown in Fig. 4, the translation error sufficiently captures the significant change in combustion state with decreasing the equivalence ratio. This indicates that the translation error has a possibility to be used for detecting the precursor of lean blowout by setting a threshold.

The standard deviation of the pressure fluctuations $p^{\prime}{ }_{r m s}$ is shown in Fig. 5 as a function of the equivalence ratio $\phi . p_{r m s}^{\prime}$ at $\phi=0.70$ gradually decreases with decreasing $\phi . \quad p_{r m s}^{\prime}$ drastically decreases when the intermittency of the pressure fluctuations occurs. With a further decrease in $\phi$, it monotonically decreases. 


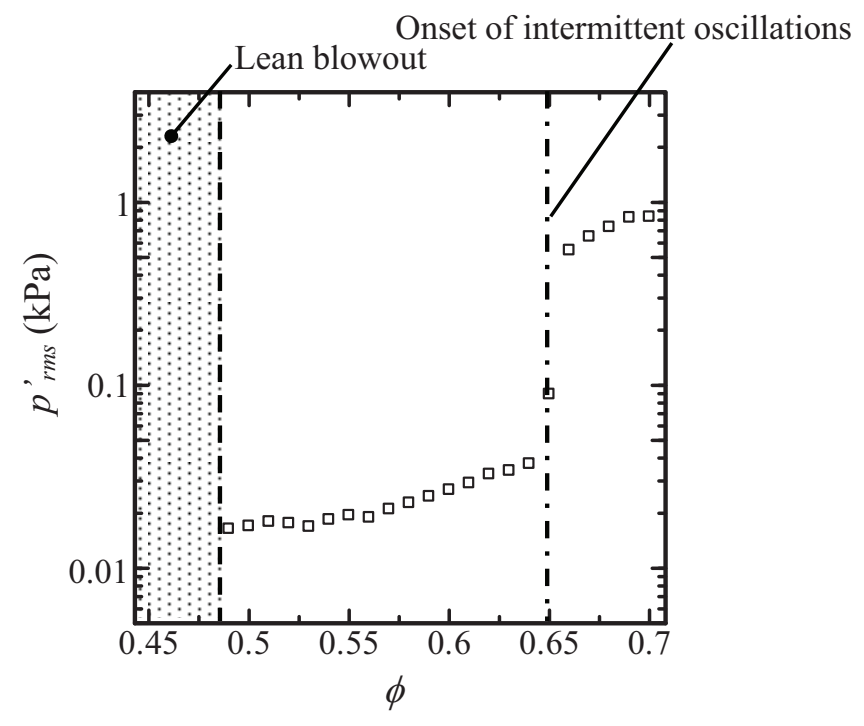

Figure 5: $\quad p_{r m s}^{\prime}$ as a function of $\phi$.

However, it remains nearly unchanged for $\phi \leq 0.56$, which means that the standard deviation of the pressure fluctuations cannot capture the change of combustion state close to lean blowout. This indicates that it is very difficult to determine the appropriate threshold of the standard deviation of the pressure fluctuations for detecting the lean blowout. These results clearly show that the translation error we applied in this work can characterize the combustion state near lean blowout and has a potential use for detecting the lean blowout in the lean premixed gas-turbine model combustor. In this work, we focused on investigating the feasibility of the translation error as a detector from the practical viewpoint. In our future work, we will introduce a control system similar to the previous study [21] to detect the precursor of lean blowout by utilizing the translation error, and also show that our method is capable of preventing lean blowout.

\section{Summary}

We have proposed a detection method of lean blowout in a laboratory-scale premixed gas-turbine model combustor from a view point of nonlinear dynamics. The translation error, which quantifies the degree of the parallelism of trajectories in the phase space constructed from the time-series data of the combustion-chamber pressure fluctuations, was used as a detector in this work. It was shown that the onset of the intermittency of the pressure fluctuations can be detected by the proposed method. The determinism in the dynamic behavior of the pressure fluctuations was lost close to lean blowout, but its dynamic behavior was successfully characterized by the translation error. The obtained 
results show that the translation error has a potential use for detecting the lean blowout in a lean premixed gas-turbine combustor.

\section{Acknowledgements}

One of the authors (H.G.) was partially supported by a "Research Grant from Paloma Environmental Technology Foundation" and "Grant-in-Aid for Young Scientists (A) from the Ministry of Education, Culture, Sports, Science and Technology of Japan (MEXT)"

\section{References}

[1] Huang, Y. and Yang, V., Dynamics and stability of lean-premixed swirlstabilized combustion, Progress in Energy and Combustion Science, 35, p. 293-364, 2009.

[2] Candel. S., Combustion dynamics and control: Progress and Challenges, Proceedings of the Combust Institute, 29, p. 1-28, 2002.

[3] Poinsot, T., Trouve, A., Veynante, D., Candel, S. and Esposito, E., Vortexdriven acoustically coupled combustion instabilities, Journal of Fluid Mechanics, 177, p.265-292, 1987.

[4] Schadow, K. C. and Gutmark, E. J., Combustion instability related to vortex shedding in dump combustors and their passive control, Progress in Energy and Combustion Science, 18, p.117-132, 1992.

[5] Shih, W. P., Lee, J. G. and Santavicca, D. A., Stability and emissions characteristics of a lean premixed gas turbine combustor, Proceedings of the Combustion Institute, 26, p. 2771-2778, 1996.

[6] Lieuwen, T. C., Experimental investigation of limit cycle oscillations in an unstable gas turbine combustor, Journal of Propulsion and Power, 18, p. 6167, 2002.

[7] Dowling, A. P. and Morgans, A. S., Feedback control of combustion oscillations, Annual Review Fluid Mechanics, 37, p. 151-182, 2005.

[8] Tachibana, S., Zimmer, L., Kurosawa, Y. and Suzuki, K., Active control of combustion-driven oscillations by secondary fuel injection, Nagare, 25, p. 219-227, 2006.

[9] Tachibana, S., Kurosawa, Y. and Suzuki, K., Active control of combustion oscillations in al lean premixed combustor by secondary fuel injection coupling with chemiluminescence imaging technique, Proceedings of the Combustion Institute, 31, p. 3225-3233, 2007.

[10] Daw, C. S., Thomas, J. F., Richards, G. A. and Narayanaswami, L. L., Chaos in thermal pulse combustion, Chaos, 5, p. 662-670, 1995.

[11] T. Kaminski, M. Wendeker, K. Urbanowicz, G. Litak, Combustion process in a spark ignition engine: dynamics and noise level estimation, Chaos, 14, p. 461-466, 2004.

[12] Sen, A. K., Litak, G., Kaminski, T. and Wendeker, M., Multifractal and statistical analyses of heat release fluctuations in a spark ignition engine, Chaos, 18, 033115(6 pages), 2008. 
[13] Gotoda, H., Miyano, T. and Shepherd, I. G., Dynamic properties of unstable motion of swirling premixed flame generated by a change in gravitational orientation, Physical Review E, 81, 026211(10 pages), 2010.

[14] Kabiraj, L., Sujith, R. I. and Wahi, P., Bifurcations of self-excited ducted laminar premixed flames, Journal of Engineering for Gas Turbines and Power, 134, 031502(7 pages), 2012.

[15] Noble, A. C., King, G. B., Laurendeau, N. M., Gord, J. R. and Roy, S., Nonlinear thermoacoustic instability dynamics in a Rijke tube, Combustion Science and Technology, 184, p. 293-322, 2012.

[16] Grassberger, P. and Procaccia, I., Characterization of strange attractors, Physical Review Letters, 50, p. 346-349, 1983.

[17] Gotoda, H., Nikimoto, H., Miyano, T. and Tachibana, S., Dynamic properties of combustion instability in a lean premixed gas-turbine combustor, Chaos, 20, 013124(11 pages), 2011.

[18] Takens, F., Dynamical systems in Turbulence, Lecture Notes in Mathematics, vol. 898, Springer-Verlag, 1981.

[19] Wayland, R., Bromley, D., Pickett, D. and Passamante, A., Recognizing determinism in a time series, Physical Review Letters, 70, p. 580-582, 1993.

[20] Gotoda, H., Amano, M., Miyano, T., Ikawa, T., Maki, K. and Tachibana, S., Characterization of complexities in combustion instability in a lean premixed gas-turbine model combustor (to be submitted).

[21] Li, H., Zhou, X., Jeffries, J. B. and Hanson, R. K., Active control of lean blowout in a swirl-stabilized combustor using a tunable diode laser, Proceedings of the Combustion Institute, 31, p. 3215-3223, 2007. 